\title{
Correction to: Observation of submesoscale turbulence in a cyclonic eddy
}

\author{
Yongchui Zhang ${ }^{1,2} \cdot$ Changming Dong ${ }^{2,3} \cdot$ Xi Chen $^{1} \cdot$ Yang Wang ${ }^{4,5}$
}

Published online: 22 February 2020

(C) Springer-Verlag GmbH Germany, part of Springer Nature 2020

\section{Correction to: Ocean Dynamics https://doi.org/10.1007/s10236-020-01349-5}

The original version of this article unfortunately contained a mistake. During the production process, Fig. 4 was presented incorrectly which was the same as Fig. 5. The corrected figure is given here.

The original article has been corrected.

The online version of the original article can be found at https://oi.org/ 10.1007/s10236-020-01349-5

\section{Xi Chen}

lgdxchxtemp@163.com

1 College of Meteorology and Oceanography, National University of Defense Technology, Nanjing 211101, China

2 Southern Marine Science and Engineering Guangdong Laboratory (Zhuhai), Zhuhai 519082, China

3 Oceanic Modeling and Observation Laboratory, Marine Science College, Nanjing University of Information Science and Technology, Nanjing 210044, China

4 School of Marine Science and Technology, Northwestern Polytechnic University, Xi'an 710072, China

5 Maritime Environment Construction Office, Beijing 100061, China 

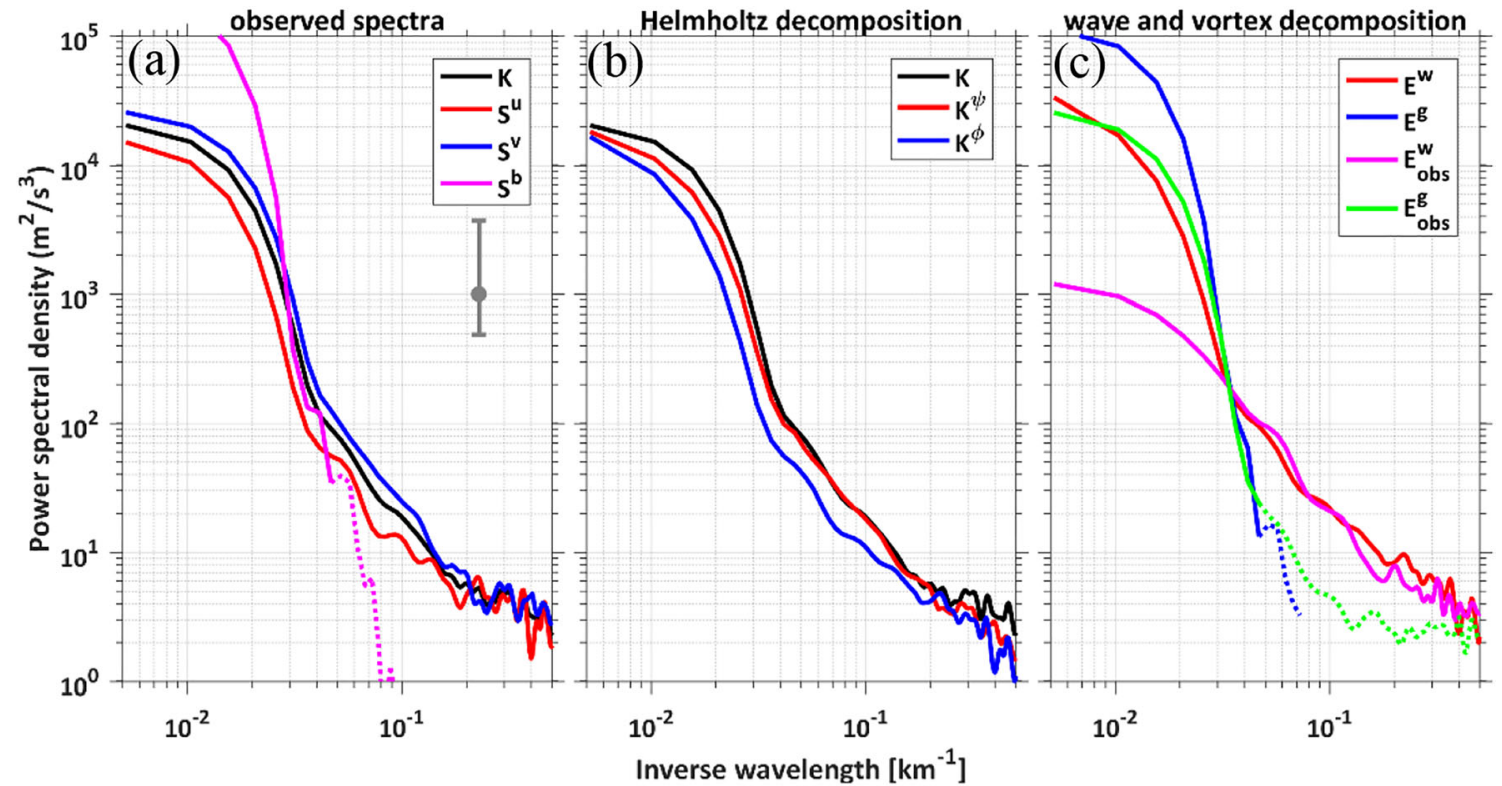

Fig. 4 Observed 6 zonal tracks average spectra and their decomposition at 48-m depth. a Observed kinetic energy and potential energy spectrum. The black, red, blue, and magenta lines are the spectra of kinetic energy $K(k)$, longitudinal kinetic energy $S^{u}(k)$, transverse kinetic energy $S^{v}(k)$, and potential energy $S^{b}(k)$. The dashed magenta line shows $S^{b}(k)$ with wavenumbers larger than distinguishable resolution. The error bar represents $95 \%$ confidence limits. b The Helmholtz decomposition. The black, red, and blue lines are the spectra of observed kinetic energy $K(k)$ into its

rotational $K^{\varphi}(k)$ and divergent $K^{\phi}(k)$ components. c Wave-vortex decomposition. The red and blue lines are the spectra of inertia-gravity wave component $E^{w}(k)$ and the residual geostrophic component $E^{g}(k)$. Observed geostrophic energy $E_{\text {obs }}^{g}(k)$ (magenta line) is calculated by temperature-salinity and residual inertia-gravity wave component $E_{o b s}^{w}$ (k) (green line). The dashed lines show wavenumber greater than maximum distinguishable resolution 